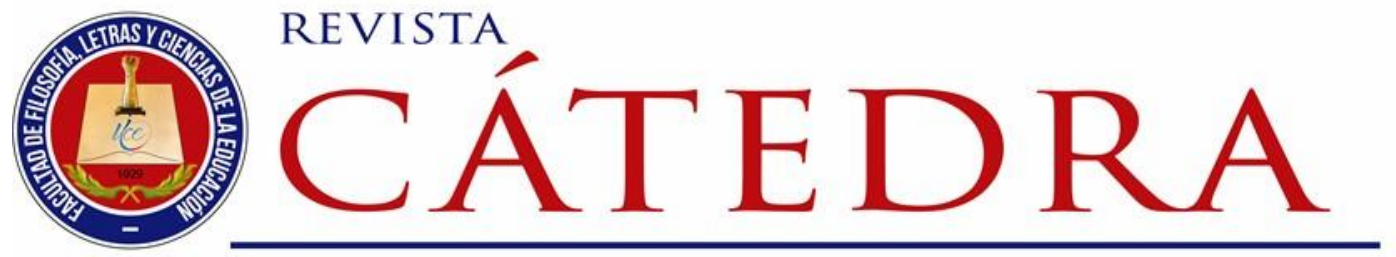

\title{
Enfoques innovadores de educación ambiental con el aprovechamiento de residuos orgánicos urbanos
}

\section{Innovative approaches of the environmental education with the use of urban organic residues}

\author{
Alisson Yépez-Chávez \\ Universidad UTE, Quito, Ecuador \\ ycav104074@ute.edu.ec \\ https://orcid.org/0000-0003-1541-943x \\ Fausto Viteri-Moya \\ Universidad UTE, Quito, Ecuador \\ faustor.viteri@ute.edu.ec \\ https://orcid.org/0000-0002-1458-8920
}

(Recibido: 06/03/2019; Aceptado: 19/03/2019; Versión final recibida: 03/04/2019)

Cita del artículo: Yépez-Chávez A. y Viteri-Moya F. (2019). Enfoques innovadores de educación ambiental con el aprovechamiento de residuos orgánicos urbanos. Revista Cátedra, 2(2), 111- 132.

\section{Resumen}

El presente documento nace de la necesidad de fortalecer la educación ambiental en el ámbito educativo a través del aprovechamiento de residuos orgánicos, que son vistos como desechos y generadores de problemas ambientales, económicos y sociales. Se determina la importancia de la educación ambiental como herramienta esencial para concientizar y sensibilizar, sobre todo para lograr el empoderamiento de los estudiantes y fomentar una cultura ecológica. El presente artículo tiene como propósito analizar las oportunidades de educación ambiental enfocadas en el aprovechamiento de residuos orgánicos generados a nivel urbano. Evaluar la educación ambiental formal en diferentes grupos objetivo y la relación de su aprendizaje con las TIC (Tecnologías de Información y Comunicación) y redes sociales. Su principal aporte es una presentación de la realidad de la educación ambiental 
en el país y en diferentes contextos culturales. La metodología utilizada en el estudio se apoya en la investigación bibliográfica, de tipo expositiva; en donde además se recopila información sobre las falencias y proyectos educativos en el Ecuador en temas ambientales.

Palabras clave

Ambiente, aprovechamiento, educación, residuos orgánicos, TIC.

\section{Abstract}

This document arises from the need to strengthen environmental education in the educational field through the use of organic waste, which are seen as waste and generators of environmental, economic and social problems. The importance of environmental education as an essential tool to raise awareness and sensitize, especially to achieve the empowerment of students and promote an ecological culture. The purpose of this article is to analyze the opportunities for environmental education focused on the use of organic waste generated at the urban level. Evaluate formal environmental education in different target groups and the relationship of their learning with ICT (Information and Communication Technologies) and social networks. Its main contribution is a presentation of the reality of environmental education in the country and in different cultural contexts. The methodology used in the study is based on bibliographical research, of an expository nature; In addition, information is collected on the shortcomings and educational projects in Ecuador on environmental issues.

\section{Keywords}

Environment, use, education, organic waste, ICT

\section{Introducción}

La generación de basura es uno de los problemas más importantes en la sociedad, tiene relación con su crecimiento y sus actividades. Los residuos son directamente proporcionales al aumento del nivel económico de un país. La cultura del desecho prima en el medio y se manifiesta a través de las siguientes cifras obtenidas del INEC (Instituto Nacional de Estadísticas y Censos) (2016) que expresan: "Según la caracterización de basura realizada en 2016, el 58\% de residuos corresponde al componente orgánico y el $42 \%$ a residuos inorgánicos (cartón, papel, plástico, vidrio, madera, metal, chatarra, caucho, textil, focos, pilas y desechos sanitarios no peligrosos, entre otros)" (p.15).

En lo citado anteriormente, se puede observar que los datos del componente orgánico son altos. Esto puede ocasionar contaminación al suelo, agua y aire, además de una afectación a la salud humana. Por ello, es primordial vincular a la sociedad para tener como resultado: decisión, acción, sensibilización y mejora de la dinámica del ciclo de vida de la basura. Con ello, se puede evitar centrarse en soluciones o alternativas de fin de tubo, que encubren la contaminación o la transfieren a otro medio. Es decir, se habla de medidas reactivas y de almacenamiento de la basura, en donde su aprovechamiento no está contemplado.

En lo que respecta a la clasificación de basura, ésta ha sufrido un descenso en los hogares ecuatorianos, es así que según el INEC (2017a):

En relación con la proporción de hogares que no clasificaron sus residuos, entre las ciudades auto representadas se verifica una tendencia de descenso, siendo el mayor de ellos el registrado en Quito con una 
reducción del 9.71\%, seguido por Machala7.31\% y Guayaquil 3.24\%. (p.

$6)$.

Con este antecedente se puede afirmar que existe una disminución de conciencia ambiental en las principales ciudades del país. Esto puede deberse a la ausencia de información sobre cómo y en dónde disponer los residuos, además por la falta de interés en los hogares en cuanto a temas ambientales.

El escenario de la basura en el país se basa principalmente en la generación, recolección y disposición. El aprovechamiento de residuos y su reutilización se encuentran en segundo plano. Pues, en muchos lugares, no existe una debida clasificación de la basura desde su origen. Esto, dificulta obtener materiales de interés, debido a que se encuentran mezclados con el resto de basura y al ser de difícil separación, pueden perder su valor y sus características.

Los residuos orgánicos pueden ser vistos como un recurso, al ser aprovechados en distintas aplicaciones. El compostaje es una de ellas, consiste en un abono orgánico a base de residuos vegetales que mejoran las propiedades y nutrición del suelo. Es una opción viable y económica, que puede ser utilizada en cultivos destinados para la alimentación o la ornamentación (Vargas et al., 2011, pp. 39-40). Otro tipo de abono es el lombricompuesto, que se produce a través de la acción de la lombriz roja californiana en residuos orgánicos (EMGIRS EP, 2017).

El uso en la incineración es otra alternativa para los residuos sólidos orgánicos urbanos (RSOU), proceso mediante el cual se puede obtener energía eléctrica. Calvachi y Navarro (2013) realizaron un estudio del poder calorífico de los RSOU en Ecuador, cuyos resultados muestran un intervalo entre $15.86 \mathrm{KJ} / \mathrm{g}$ a $17.08 \mathrm{KJ} / \mathrm{g}$ (p. 98). Esto demuestra su viabilidad para la incineración, pues según la ISWA (International Solid Waste Association, 2013) el poder calorífico de los residuos debe ser como mínimo $7 \mathrm{KJ} / \mathrm{g}$ para tener eficiencia (p.5).

A partir de ello, también se puede obtener biogás, que es resultado de la degradación anaerobia de la materia orgánica, esto puede ser utilizado para la calefacción, transporte, generación de electricidad, etc. La producción de biogás de forma controlada, permite la disminución de gases de efecto invernadero (GEI). A razón de que, en la combustión, el metano se transforma en dos productos: $\mathrm{CO}_{2}$ y agua, evitando su difusión directa hacia la atmósfera, como se muestra en la Ecuación 1. Esto es una ventaja, puesto que el metano de forma incontrolada produce mayor contaminación que el $\mathrm{CO}_{2}$ (Romero, 2010, p. 344).

$$
\begin{gathered}
\mathrm{CH}_{4}+2 \mathrm{O}_{2} \rightarrow 2 \mathrm{H}_{2} \mathrm{O}+\mathrm{CO}_{2} \\
\text { Ecuación } 1
\end{gathered}
$$

En este contexto, el biodiésel y bioetanol son biocombustibles, cuya materia prima también puede provenir directamente de cultivos o a partir de residuos. Su aplicación se enfoca principalmente en el transporte. Presentan distintas ventajas: disminuyen las emisiones de GEI, principalmente $\mathrm{CO}_{2}$ hasta un 50\%. De acuerdo con Jaramillo y Zapata (2008) "Los biocombustibles son biodegradables, el $85 \%$ se degrada en aproximadamente 28 días" (p. 45). Con esta apreciación, se resalta la importancia del uso de biocombustibles, pues su descomposición se genera en condiciones naturales ocasionando menor impacto al medio.

Al analizar el ciclo de producción de los biocombustibles se puede comparar la manera de obtenerlos, puesto que, si se lo hace a través de cultivos alimenticios como maíz o caña de azúcar, podría vulnerar la seguridad nutricional y ambiental. Por otra parte, si su producción es a partir de materiales sin ningún valor como la basura, desechos orgánicos,

Licencia Creative Commons Atribución 4.0 Internacional (CC BY 4.0)

Revista Cátedra, 2 (2), pp. 111-132, mayo-agosto 2019. e-ISSN: 2631-2875

https://doi.org/10.29166/catedra.v2i2.1639 
esto significaría una menor contaminación, disminución de desechos y reducción de los costos que se generan en su disposición.

En el ámbito educativo, Ecuador cuenta con una Estrategia Nacional de Educación Ambiental para el año 2017-2030. Su objetivo es vincular a las instituciones públicas, privadas (en niveles iniciales, básico y superior) y ciudadanía en la sostenibilidad. Es decir, se habla de un proceso extenso que vincula a otros sistemas: económico, político y social.

Como resultado, según la página web del MAE (Ministerio del Ambiente, 2017), este plan arrojó que no se ha logrado llegar a un nivel alto en optimización de recursos e investigación. Existen deficiencias en la clasificación de residuos y en la formación docente en temas ambientales. Otra de las falencias identificadas se encuentra en las Instituciones de Educación Superior (IES), en donde más del 50\% de ellas cuentan con política ambiental. Sin embargo, en base al porcentaje mencionado solo el $44 \%$ la aplican adecuadamente.

Otro reciente programa educativo es el denominado: "Tierra de niños y jóvenes" (TINI), emprendido por el Ministerio de Educación del Ecuador desde 2017. Tiene como propósito crear cultura y conciencia ambiental en las instituciones educativas. Proporciona un espacio físico tanto a jóvenes como niños, en el que serán actores principales en el cuidado y desarrollo de vida y biodiversidad.

Los jóvenes y niños interactúan con el medio a través de actividades como cuidado, siembra de plantas y árboles, construcción de refugios para aves, etc. Dentro de los principales componentes utilizados para la implementación del programa se encuentran el manejo de recursos naturales, salud ambiental, socialización, expresión, organización y gestión. Cabe destacar que dentro del componente referente a salud ambiental, sus actividades se enfocan en la segregación de los residuos sólidos, además de su aprovechamiento para la elaboración de abonos orgánicos (Ministerio de Educación, 2016, pp. 11-19). A pesar de los esfuerzos gubernamentales, la cultura ambiental no ha alcanzado los resultados esperados. Se debe trabajar en programas dinámicos, de acuerdo a grupos específicos. A continuación, se hablará de la educación ambiental, sus características, enfoques y rasgos relevantes.

La educación ambiental (EA) debe ser integral, relacionada con los sistemas socioculturales, económicos, tecnológicos, políticos y ambientales. Es decir, se cambia la perspectiva del hombre y la naturaleza, como lo manifiesta Novo (2009) con una nueva visión ideológica en la que "Contempla al ser humano no como dominador o dueño de la naturaleza sino como parte de ella, como una especie que, con sus indudables singularidades, está retada a entenderse y desarrollarse en armonía con el resto de la biosfera" (p. 202). Esto hace hincapié en la influencia que tiene la naturaleza en nosotros y que el estudio de lo que nos rodea representa el conocimiento propio. Formamos parte de un sistema, constituido de relaciones y elementos que conforman un todo y por lo tanto cualquier decisión va a afectar a ese todo.

De acuerdo a Castillo (2010) en su artículo sobre la importancia de la EA como problemática actual, se establece que el aprendizaje se fundamenta en la creación de experiencias, conocimientos y valores que trascienden a lo largo del tiempo. No se busca la memorización, por el contrario, se busca la participación activa para asegurar la permanencia de conciencia y que ésta se convierta en un hábito. Una orientación ética posibilita el nexo para la sustentabilidad, a través del cambio de actitudes y consideraciones hacia los demás. Todo esto da como resultado la posibilidad de lograr un pensamiento crítico y mejor resolución de problemas (Castillo, 2010, pp. 105-108).

Licencia Creative Commons Atribución 4.0 Internacional (CC BY 4.0)

Revista Cátedra, 2 (2), pp. 111-132, mayo-agosto 2019. e-ISSN: 2631-2875

https://doi.org/10.29166/catedra.v2i2.1639 
Todo lo acotado anteriormente hace que la educación ambiental sea un eje primordial para el desarrollo de nuevas metodologías en la utilización de residuos, siendo ésta una oportunidad para que las nuevas generaciones conozcan y tomen acción frente a problemas cotidianos como la acumulación de residuos. Antes considerados meros desechos, sin valor comercial, y que actualmente son vistos como materia prima para su futuro aprovechamiento.

El presente artículo abordará cuatro secciones principales: educación ambiental por grupos objetivo; la influencia que tienen las TIC y redes sociales en la educación ambiental y las oportunidades de educación ambiental en el aprovechamiento de residuos orgánicos.

\section{Educación ambiental por grupos objetivo}

\subsection{Enfoque de edades}

En el estudio de Pol y Castrechini (2013) realizado en Cataluña, se desarrolló una evaluación a niños, jóvenes y adultos en edades entre 9 y 35 años para determinar su conducta ecológica. Se concluye que, la EA posee mayor efectividad en los grupos de edades entre 8 y 13 años, pues presentan puntuaciones más altas que significan un mayor conocimiento, conciencia y valores ambientales. Por otro lado, la conciencia ambiental en la adolescencia sufre una disminución que se reintegra nuevamente en la etapa adulta.

Con el pasar del tiempo, se pierde la conciencia ambiental adquirida en edad temprana. La ausencia de programas ambientales muchas veces no es la causa; tiene que ver con los procesos y cambios psicológicos que experimentan los adolescentes. La rebeldía hacia normas establecidas y la falta de concordancia entre la comunicación verbal y las acciones en autoridades, fomentan la deficiencia de compromiso hacia temas ambientales. Esto se evidencia a través de la Figura 1.

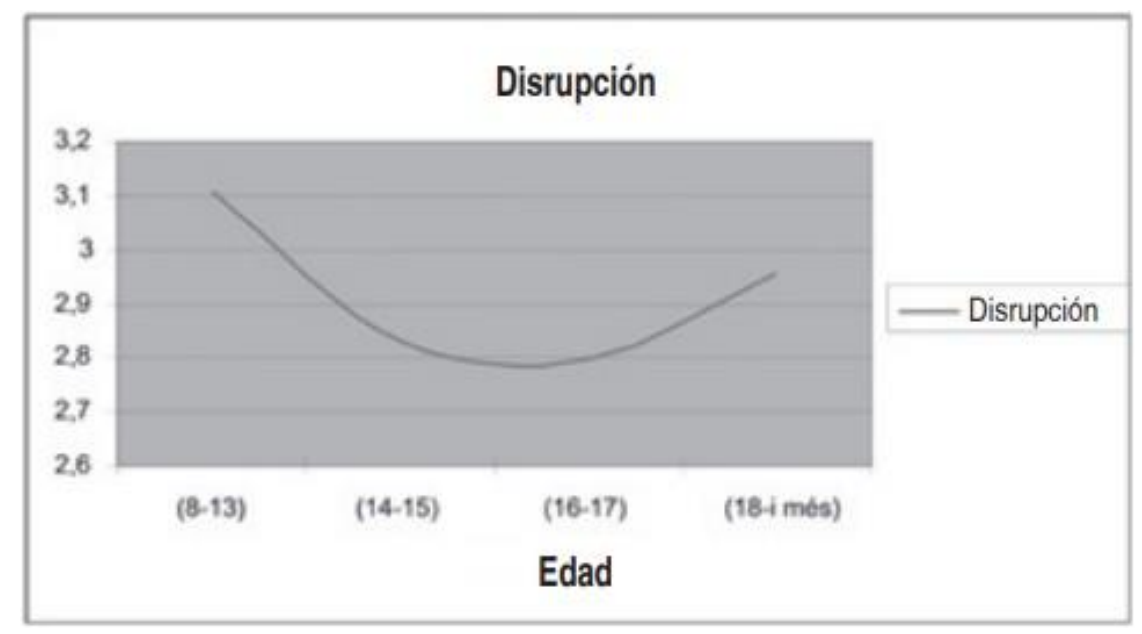

Figura 1. Disrupciones en la EA. Fuente: (Pol y Castrechini, 2013, p. 341).

Como se muestra en la Figura 1, en las edades correspondientes a la infancia, desde los 8 a 13 años, existen puntuaciones altas en cuanto a conocimientos y valores ambientales. A partir de los 14 a 17 años, existe un notable descenso, una disrupción muy marcada. Sin embargo, en la edad adulta esta conciencia ambiental aumenta nuevamente, pero no consigue igualar a las puntuaciones de periodos iniciales.

Los niños se encuentran en una etapa de formación tanto en nivel educativo como en valores. Son más perceptivos en cuanto al nuevo conocimiento. Se encuentran

Licencia Creative Commons Atribución 4.0 Internacional (CC BY 4.0)

Revista Cátedra, 2 (2), pp. 111-132, mayo-agosto 2019. e-ISSN: 2631-2875

https://doi.org/10.29166/catedra.v2i2.1639 
condicionados a seguir con los parámetros que se les establecen. Es decir, se habla de un mayor control hacia ellos y se puede generar mayor influencia en sus hábitos.

La disminución de conciencia ambiental en adolescentes se ve reflejada en otro estudio realizado por Boeve et al., (2015), en donde participan estudiantes entre 12 a 19 años en 51 escuelas de Suecia. Se reafirma que en "edades hasta los 13 años, existe una rápida incorporación de conceptos y actitudes ambientales. Mientras que, en edades superiores la motivación y el interés sufre un significativo descenso" (pp. 15708-15711). Lo mismo sucede en otro estudio realizado por Olsson y Gericke (2015), en estudiantes entre 12 a 19 años en escuelas de Suecia. En donde se establece que "existe una decadencia en la eficiencia de EA, en la adolescencia. También se constata que no existe un declive total en la EA, pues en la etapa madura vuelve a ascender" (pp. 41-42).

Lo mencionado anteriormente, puede ser causado por los problemas de esta etapa de transición, que se caracterizan por la constante lucha interior, clave para la definición de la personalidad y valores. Se construyen relaciones interpersonales e intrapersonales. Se desarrollan constantes conflictos de poder entre los adultos, debido a la diferencia de pensamientos y a la búsqueda de su identidad. El grupo social tiene mayor influencia que las personas adultas. Su pensamiento se centra en su individualidad y en el presente, su principal preocupación es su vida sentimental y social (Garza, 2003, pp. 36-38).

En la etapa universitaria se destaca la falta de implicación en los problemas ambientales. Las personas no se sienten parte de la solución, suprimiendo su responsabilidad y aceptación de las consecuencias que traen consigo la destrucción del medio. La influencia social determina el comportamiento ambiental de las personas, por lo general estas acciones sirven como parámetro de comportamiento (Pol y Castrechini, 2013, pp. 345-346).

Además, en la EA se recomienda aplicar distintas estrategias acordes al nivel de madurez y edades, como lo contempla Corraliza (2016) quien manifiesta que existen 4 fases para la EA. La primera fase consiste en desarrollar sentimientos positivos, a través de una aproximación física y visual del entorno. La segunda fase hace hincapié a la prevención y al inicio de la práctica para la creación de costumbres y experiencias. La tercera fase está dedicada a niños mayores de 12 años, con una visión proactiva y consciente, localizando razones personales para obrar a favor del medio. La cuarta fase, pretende instaurar un panorama sistémico, en relación a diferentes contextos tanto sociales como intrapersonales.

En la tesis de Díaz et al. (2014) se hace referencia a la EA, en la primera infancia con edades entre 3 y 5 años en el Bajo Cauca, departamento de Antioquia, Colombia. Los procesos cognoscitivos en esta fase, están influenciados por el vínculo que pueda establecerse con ellos y la naturaleza.

En cuanto a la comprensión ambiental de los niños, estos distinguen sus componentes a través de sus sentidos, de forma física y visual. Tienen ideas sobre el cuidado del medio ambiente, pero poseen una concepción de tipo naturalista, en donde el ser humano no es parte de ella, es decir, se sienten ajenos a la naturaleza.

La formación de los niños, engloba una percepción de sistemas extensos, no inmediatos al ser humano como selvas, parques, ecosistemas acuáticos, terrestres, etc. El sentido de la palabra ambiental comprende también sistemas en los que habitamos como nuestro hogar, la escuela, trabajo, las calles, nuestra ciudad. Con la comprensión de estos aspectos, se puede fomentar la acción directa de conductas ecológicas, visibles en el entorno en que los niños se desenvuelven.

Licencia Creative Commons Atribución 4.0 Internacional (CC BY 4.0)

Revista Cátedra, 2 (2), pp. 111-132, mayo-agosto 2019. e-ISSN: 2631-2875

https://doi.org/10.29166/catedra.v2i2.1639 
En el estudio de Vázquez y Manassero (2005), se examina la actuación hacia el medio ambiente en 774 estudiantes de último curso de educación secundaria a nivel internacional. Con edades comprendidas entre los 14 a 16 años; de los cuales el 57\% son mujeres. En general presentan una actitud moderada, impulsada principalmente por la idea de sustentabilidad, responsabilidad y defensa de los animales (pp. 315-316).

Los estudiantes expresan su compromiso con el cuidado ambiental, pero se desarrollan criterios negativos de externalidad hacia los problemas ambientales en ellos. Existe una subestimación de los riesgos que pueden producirse. Se habla de la participación especializada para reparar daños ambientales, como único recurso para resolver conflictos. En cierta forma, es necesario el asesoramiento científico y tecnológico, pero también es necesaria la participación de todos.

Se observa que se tiene un concepto de cambio y conservación, pero existe una actitud pasiva. Se tiene conocimiento del problema, pero no se materializa esa conciencia ambiental. Se culpa o se atribuye la contaminación a las grandes potencias, al igual que su solución.

Por otro lado, en otro estudio realizado en Chile, a estudiantes entre 14 y 16 años, se concluye que los mismos, tienen la intención de ayudar al planeta en gran medida, Así Gädicke et al., (2017), afirman que "Los estudiantes entre 14 y 16 años han realizado actividades como ahorro de energía y agua (70\%), reducción de uso de automóviles (30.8\%), clasificación de residuos (24\%)" (p. 114). Sin embargo, la constancia no se encuentra estimada en sus acciones, pudieron haberlo llevado a la práctica por periodos cortos de tiempo, para nuevamente volver a las prácticas consumistas

La EA debe iniciarse en edades tempranas, en las etapas de niñez y adolescencia, pues allí se puede lograr la construcción de un vínculo con el entorno. En primera instancia a través de percepciones sensoriales y de un reconocimiento exploratorio de lo que les rodea. Posteriormente fomentar un pensamiento crítico y reflexivo, en un espacio de confianza y comunicación. Lo que se pretende es fortalecer los primeros pensamientos y experiencias obtenidas, llevados de la mano con un crecimiento personal.

En la EA, no existe una edad correcta para su iniciación, Corraliza (2016) expresa que la EA no tiene edad. Esto debido a que desde que nacemos ya estamos en contacto con la naturaleza, que es nuestro hogar, fuente de vida y sentimientos. Por lo tanto, el conocimiento y preservación del medio se convierte en una necesidad, debido al papel que desempeña en nuestro desarrollo, para poder aprovecharlo de forma responsable y sea también fuente de disfrute.

\subsection{Enfoque de género}

Las percepciones difieren en el género, las mujeres tienen cualidades más ambientalistas. El género masculino tiene una actitud impersonal del entorno, una minimización de efectos y una confianza total en la rama científica y en los países desarrollados. Tienen mayor convicción en las grandes potencias como alternativas para la preservación. En cambio, las mujeres ven como medida la vinculación de todos los elementos que se ven involucrados en el problema Este análisis se refleja en el estudio comparativo internacional de Vázquez y Manassero (2005) con estudiantes de 14 a 16 años. (pp. 319-320)

Gädicke et al., (2017) realizó una investigación enfocada en las diferencias de género en cuanto a percepción ambiental. Participaron 452 estudiantes con edades entre 14 a 16 años, que fueron evaluados a través de una ponderación, tomando en cuenta sus percepciones frente a los problemas ambientales. Como resultado, las mujeres muestran mayor interés,

Licencia Creative Commons Atribución 4.0 Internacional (CC BY 4.0)

Revista Cátedra, 2 (2), pp. 111-132, mayo-agosto 2019. e-ISSN: 2631-2875

https://doi.org/10.29166/catedra.v2i2.1639 
preocupación, inquietud a nivel local o regional; en cuanto a problemas ambientales, principalmente sobre el cambio climático, que los hombres (pp. 115, 118, 119).

Una de las razones del papel en la percepción ambiental de la mujer es su nivel de madurez, lo cual está asociado a las características que le son impuestas por la sociedad, cuidado, solución de problemas, preocupación por los demás, etc. A nivel neuronal, en las mujeres se realiza un proceso de mejora a edad más temprana. Se optimizan los procesos mentales a través del reconocimiento de las conexiones neuronales de mayor importancia. Pero esto no quiere desacreditar la madurez de los hombres, ya que este factor se relaciona con las condiciones de vida y experiencias (Castillero, 2017).

En el estudio de-Palavecinos et al., (2016) se examinó a 237 estudiantes de España y Chile, con edades entre 18 a 26 años en Chile y 18 a 53 en España. Al contrastar el estudio en diferentes continentes, se observa que los estudiantes tienen conductas ambientalistas muy similares. Esta afirmación es interesante porque existen grandes diferencias en difusión, ejecución y disponibilidad de información y proyectos entre países. Por otra parte, los estudiantes chilenos mostraron un vínculo emocional ambiental superior, mientras que los españoles mostraron cierta indiferencia. Nuevamente, se afianza el papel de las mujeres en la lucha ambiental, ellas se reconocen como proambientalistas, tienen mayor conexión y empatía (pp. 146-148).

Existe una mayor preocupación por el hecho de que el desarrollo económico aumenta, el propósito es contribuir a mejorar el medio ambiente, como es el caso de España; pero a pesar de tener acceso a gran cantidad de información, esto no garantiza que dichas intenciones lleguen a ponerse en práctica. El enfoque cognoscitivo es importante, pero la formación emocional también, pues estos son factores que influyen en nuestra conducta. La construcción de una conexión afectiva refuerza el interés, motivación y emprendimiento de acciones favorables y estímulos emocionales son capaces de crear comportamientos más fuertes.

\section{TIC y redes sociales en la educación ambiental}

Las TIC son portales de conocimiento instantáneo, están al alcance de todos y son estratégicas a la hora de difundir información y promover una mejor interacción con los alumnos. Estas herramientas tienen gran influencia en la forma de pensar de los individuos, por lo que su manejo conlleva una gran responsabilidad.

Su importancia en el área ambiental según Carvajal (2014) es que: "Las TIC son tecnologías interdisciplinares que pueden impulsar la profunda transformación necesaria en los esfuerzos mundiales para luchar por la sustentabilidad y sostenibilidad del planeta" (p. 67) Pues, la ciencia se expresa a través de los medios, en experiencias, investigaciones, modelos, programas en distintas ramas. Pueden ser adaptados y mejorados en beneficio de una sociedad. Compartir esas experiencias, permite fortalecer la cooperación mundial hacia prácticas que generen cambios.

La era digital está muy marcada en los niños, ellos nacen aprendiendo como manejar herramientas tecnológicas. Tienen fácil acceso a ella y pueden explorar diferentes escenarios que cambian la mirada que se tiene del planeta. Pero, para ello se debe tener un criterio formado, capaz de discernir la mejor información y asimilarla adecuadamente. Estas herramientas también constituyen un problema, ya que, van acorde a tendencias sociales y poseen una obsolescencia programada que obliga a que se consuma cada vez más y más. Este es el caso de los teléfonos celulares que son herramientas habituales para la búsqueda

Licencia Creative Commons Atribución 4.0 Internacional (CC BY 4.0)

Revista Cátedra, 2 (2), pp. 111-132, mayo-agosto 2019. e-ISSN: 2631-2875

https://doi.org/10.29166/catedra.v2i2.1639 
de información, conexión y comunicación, pero se ha visto que la conexión digital debilita la conexión emocional.

El manejo de bibliotecas e información en línea refuerzan los conocimientos impartidos en el aula de clase. Se cambia de sistemas cerrados a sistemas abiertos, donde los estudiantes desarrollan por sí mismos los temas que han recibido. La curiosidad hace que se formen preguntas y necesidades de respuesta que pueden ser encontradas en estos medios en lenguaje tanto coloquial como técnico. El docente va a servir de guía, formador de criterio y apoyo para distinguir la información carente de respaldos. No se puede eliminar la función del profesor, que actúa como mediador entre los alumnos y las herramientas tecnológicas. Además, crea un espacio de discusión y tutorías para afianzar el conocimiento (Zúñiga, 2004, pp. 3941).

La tecnología tiene potencial de proactividad y diligenciamiento de acciones frente a desastres naturales. Comunica de forma inmediata y descentraliza la ayuda. Es fuente de contribución, participación e información geográfica para potenciar la prevención. El monitoreo del medio físico en tiempo real da una perspectiva de lo que sucede alrededor. Adapta a la sociedad y crea resistencia a los problemas ambientales (Johnson y Valdiviezo, 2011, p. 9).

Según cifras de la Fundación Española para la Ciencia y Tecnología (FECYT), en la Encuesta de Percepción Social de La Ciencia y La Tecnología de 2018, en España

la televisión es la fuente más usada para obtener información en un 75\%, el $40 \%$ de la población usa el internet en como primera opción para informarse. Las redes sociales son las más utilizadas en personas menores a 35 años, seguida de plataformas visuales como YouTube. Por otro lado, en Ecuador, según el INEC (2017b) el 58\% de la población mayor a 5 años utiliza el internet, de ello el $74 \%$ lo hace de forma habitual, como mínimo una vez al día. El uso de las redes sociales es masivo con un $91 \%$ (pp. 14-15; 22,41).

Como se puede observar, los medios de mayor importancia para la difusión de información son los visuales como la televisión, además el internet, que genera influencia a través de las redes sociales. Debido a su elevado uso, son herramientas con potencial para educar en conciencia ambiental.

Los medios de comunicación tienen gran capacidad de influencia. El problema radica en el tipo de información que se muestra, es de tipo amarillista, busca llamar la atención de los usuarios a través de noticias impactantes. El compromiso de los medios es transmitir información objetiva, basada en la investigación y en un lenguaje entendible para la población. Para ello, es necesaria una preparación periodística en temas ambientales, para lograr una interpretación adecuada.

Se realizó una investigación por Cervantes (2015) en Monterrey en el año 2013, en el que se estimó la cantidad información ambiental en canales de televisión, radio y prensa. Los resultados mostraron que no hay gran interés por mostrar este tipo de información, se prefiere el entretenimiento, farándula y escándalos. Existen secciones de tipo ecológicas, pero no son ampliadas, debido a su falta de interés. La autora determinó que:

De 266 veces que se abordaron temas ambientales, 208 fueron en el género de noticia que proporciona información de un hecho sin propósitos pedagógicos. Solo 15 veces se dio un tratamiento didáctico con objetivos pedagógicos. Los medios observados orientaron 66 veces

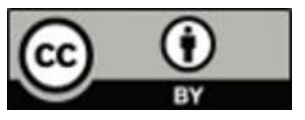

Licencia Creative Commons Atribución 4.0 Internacional (CC BY 4.0)

Revista Cátedra, 2 (2), pp. 111-132, mayo-agosto 2019. e-ISSN: 2631-2875

https://doi.org/10.29166/catedra.v2i2.1639 
la información que proporcionaban sobre medio ambiente hacia la reflexión y toma de conciencia (p. 19).

Con estas cifras se evidencia que se muestran noticias de problemas ambientales, pero no se profundiza en el accionar frente a estos. Las noticias no se enfocan en crear cultura en la población, solo en mostrar hechos de alto impacto. Muchas veces, se da un espacio para la reflexión, pero está enfocada en aspectos negativos y a nivel global. No se enfocan en el problema, sino en sus actores. Se presenta con mayor énfasis la realidad global, sin contemplar la realidad local, que forma parte del problema.

Por otro lado, las redes sociales son las más usadas por el mundo en un rango amplio de edades. La red social más utilizada es Facebook, así Ruiz (2016) enuncia que:

Facebook alcanzaba los 1.490 millones de usuarios activos en julio de 2015, y en agosto conseguía el hito de 1.000 millones de usuarios conectados el mismo día ( 1 de cada 7 habitantes del planeta). Twitter y Linkedin continúan creciendo cada año, y el uso de plataformas de fotografías y vídeos como Youtube, Instagram o Pinterest ha aumentado de manera exponencial.

Facebook es la red social más atractiva y con mayor cantidad de usuarios. Esta puede ser una herramienta de transmisión de información veraz, creativa y visual. Constituye una mezcla de las demás plataformas, ya que incluye videos, fotos, foros de opinión, grupos con diferentes intereses, etc. La información que se publica es vista como parte del ocio, informa y a la vez entretiene. La desventaja del manejo de esta red social es que cualquier individuo puede reportar mensajes que no son verificados o no están en contexto. Esto puede significar posiciones negativas sin ningún fundamento. Otros aspectos son las tendencias de temporada, acciones que no necesariamente son positivas.

Un ejemplo del uso de Facebook en la educación, se manifiesta en la investigación presentada por Acosta (2013), donde se realizó un plan piloto dirigido a estudiantes de Ingeniería Ambiental, con edades entre 16 y 21 años, tomando el asesoramiento de docentes.

El principal objetivo de la investigación fue crear un grupo en Facebook que difunda noticias generales, eventos, foros y congregaciones para investigación y describir la reacción de los estudiantes. Los docentes son elementos de vital importancia en la administración del grupo de Facebook, son una representación de credibilidad y seguridad en la información.

Las reacciones frente a la publicación de noticias no fueron positivas, con escasos comentarios y participación, los estudiantes tuvieron una predilección y mejor respuesta frente a las convocatorias para formar grupos de investigación. La publicación de videos de YouTube en esta plataforma dio como resultado gran cantidad de comentarios tanto de estudiantes como de docentes.

Se destaca el uso de Facebook por su reconocimiento mundial, fácil uso, condiciones de privacidad y seguridad, potencial de extensión de información a más usuarios especialmente jóvenes. Facebook se caracteriza por ser una plataforma dedicada al entretenimiento, esto puede dificultar la seriedad del proceso de enseñanza. Los jóvenes ven a este espacio como suyo, en donde encuentran relajación y convivencia con sus amigos. Se puede optar por redes sociales más serias, dedicadas a la opinión como Twitter (pp. 6264) 
Las TIC hacen eficiente la instrucción en los niños, pues en la investigación de Salinas et al., (2016) se evaluó el aprendizaje en niños de cuarto año de primaria en Campeche, México. Para la enseñanza se utilizaron 4 recursos: plataforma Prezi, videos interactivos, blog, elaboración de un cómic. La aceptación de estas herramientas en los niños se muestra en la Figura 2.

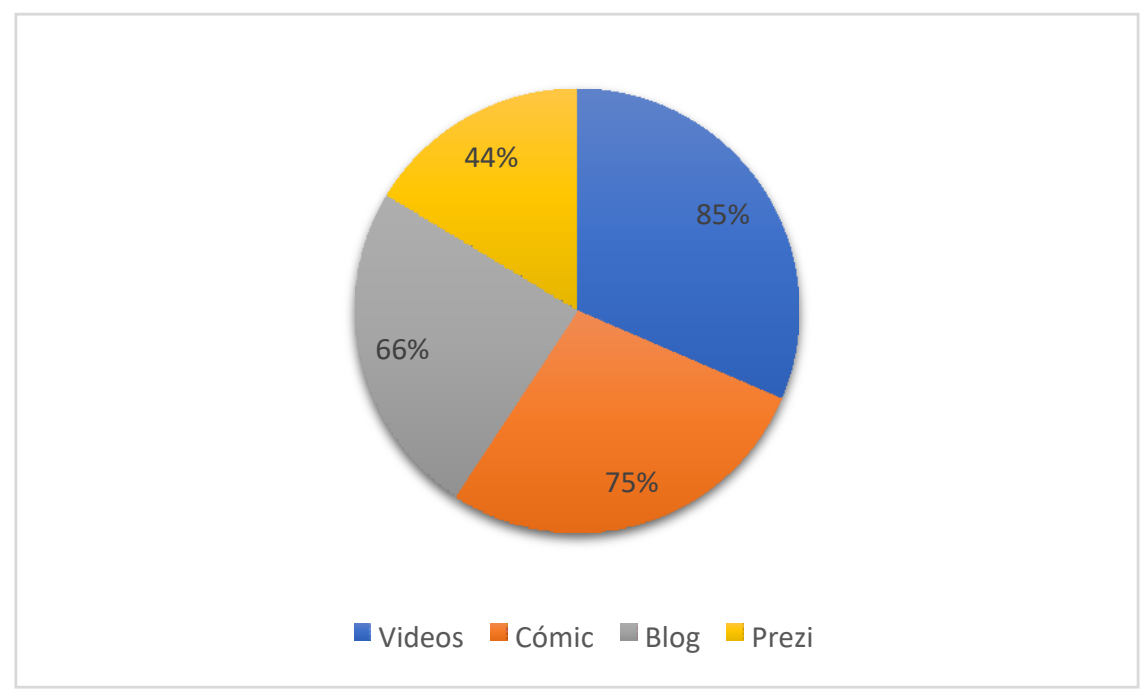

Figura 2. Aceptación de TIC en los niños. Fuente: (Salinas et al., 2016, p. 3003).

Como se puede observar las herramientas visuales son las que tienen mayor protagonismo, los videos presentados tuvieron mayor impacto y atención en los niños. Además, con el uso de estas herramientas se logró aumentar el interés ambiental de los estudiantes en un 91\% de acuerdo a encuestas realizadas.

Lo visual causa mayor impacto en los niños, llama más su atención y hace que su retención sea más eficaz. El porcentaje que no estuvo de acuerdo con estas herramientas posiblemente tenga inteligencias relacionadas a la parte kinestésica. Es por ello, que las metodologías deben estar dirigidas para varios tipos de inteligencias. El diagnóstico de los niños es imperativo para emprender con los procesos de cognición.

Los adolescentes y universitarios tienen diferentes fuentes y motivaciones para la búsqueda de información. En Hungría se realizó un estudio que comparó esos aspectos en estudiantes universitarios y de colegio, como se muestra en la Figura 3.

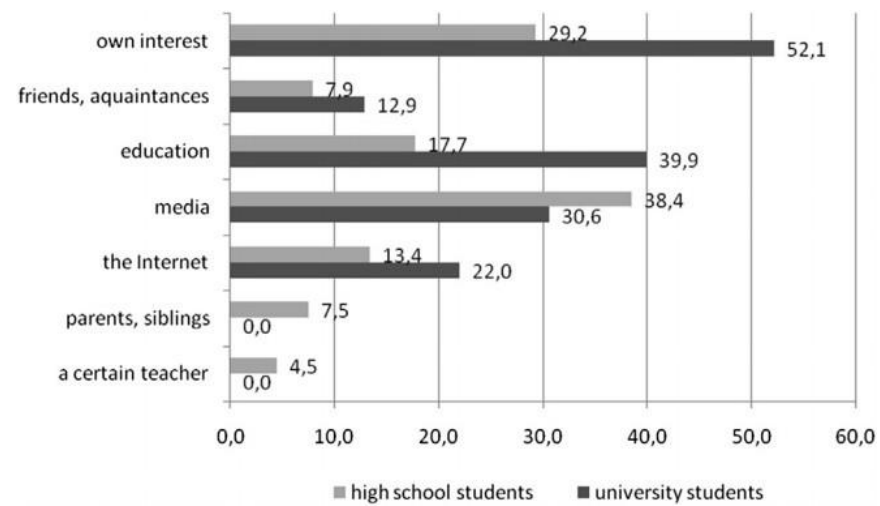

Figura 3. Fuentes y motivaciones de la EA. Fuente: (Zsóka et al., 2013, p. 129). 
Loa estudiantes de colegio poseen poco interés personal en temas ambientales, contrario a los estudiantes universitarios. El mayor instrumento de información según ambos grupos son los medios de comunicación, seguido del internet.

Para los estudiantes de colegio, la motivación para mejorar su educación es baja, mientras que para los estudiantes universitarios esta es la segunda razón que los motiva a investigar o profundizar más los temas. El papel de padres, amigos y profesores como fuente de información es menor. Este aspecto no fue considerado para la encuesta para estudiantes universitarios (Zsóka et al., 2013, p. 130).

Los estudiantes de colegio no desarrollan intereses personales en temas ambientales, esto puede deberse a que no lo consideran parte de su estilo de vida. Son inculcados en los colegios, pero son tomados en cuenta solo durante el periodo escolar. La motivación es importante, ya que nos impulsa a conocer más, tener mayor actuación frente a la problemática, sobre todo tomar mejores decisiones.

Para mejorar la asimilación de la EA en adolescentes es necesario que se combine la parte teórica y práctica en la metodología, al igual que, crear ambientes de confianza y comunicación horizontal con ejemplos, anécdotas, etc. En la investigación propuesta por Pedroso y Menéndez (2017) se resalta la educomunicación, videos, dibujos, dramatizaciones, habilidades de comunicación realizada por ellos mismos, como herramienta de aprendizaje. Todas estas actividades requieren la integración de figuras de autoridad para dar mayor fuerza y credibilidad a los proyectos. Su principal logro fue crear motivación para seguir con actividades fuera de clase y después de finalizado el proyecto. Se concluye que la mejor herramienta es la audiovisual, ya que fomenta la creatividad de los alumnos y permite que tengan una mejor percepción y tolerancia de su entorno (pp. 162164).

\section{Oportunidades de educación ambiental en el aprovechamiento de residuos orgánicos, una mirada desde otros países}

En América Latina y el Caribe, los Residuos Sólidos Urbanos (RSOU) son los principales componentes de los desechos, como se muestra en la Figura 4.

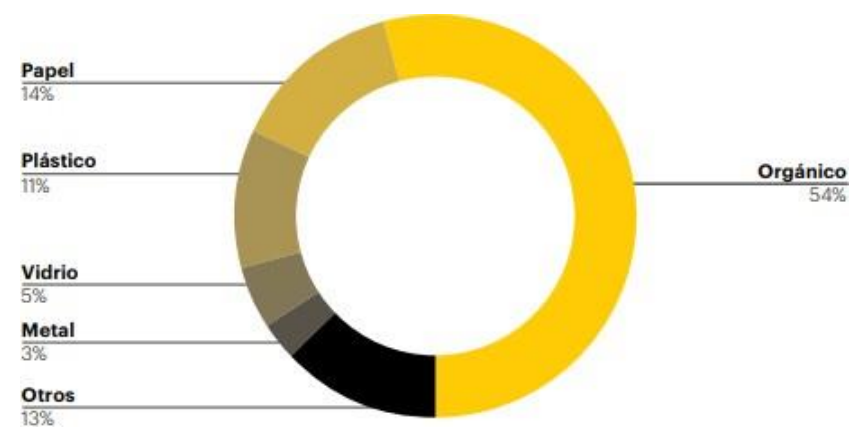

Figura 4. Composición de la basura en América Latina y el Caribe en 2012. Fuente: (Graziani, 2018, p. 14).

Los RSOU superan a los residuos inorgánicos en un 17.4\%. Los materiales inorgánicos han tomado de igual forma importancia, estos han aumentado debido al uso de tecnologías, estilo de vida más acelerado, uso de productos con vida útil reducida. Los RSOU comprenden residuos derivados de la alimentación y productos de actividades de ornamentación como: 
hojas, tallos, principalmente. En la Figura 5, se muestra la gestión de los residuos en varios países en el año 2017.

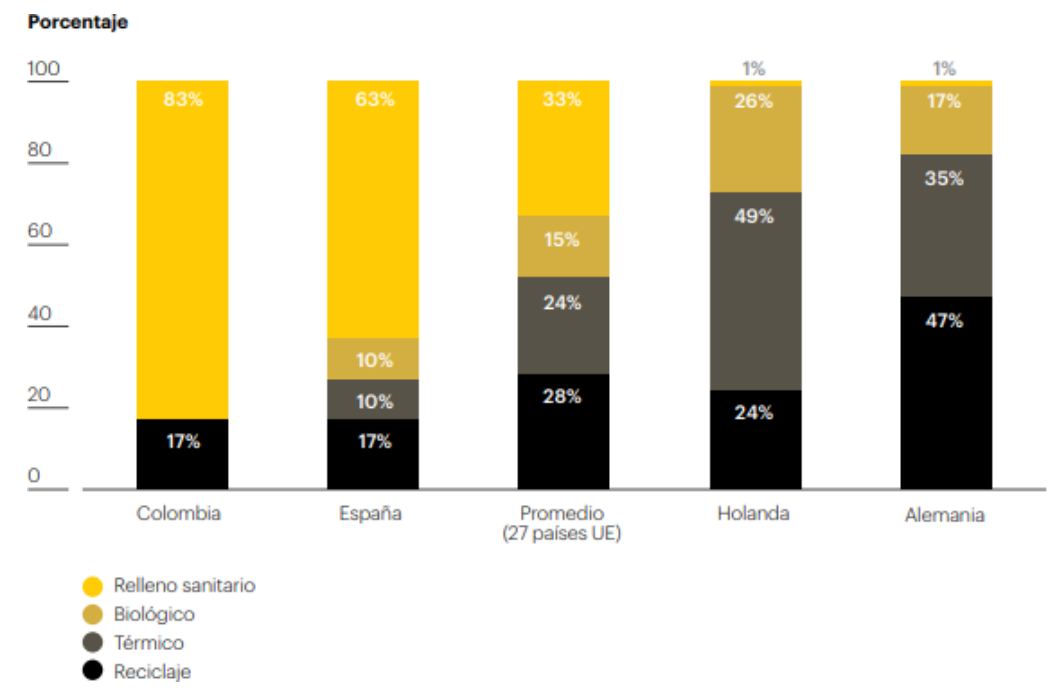

Figura 5. Gestión de los residuos en el mundo, año 2017. Fuente: (Graziani, 2018, p. 20).

En la figura anterior, se muestra la realidad de países en desarrollo en comparación con los más desarrollados. Se evidencian las falencias tecnológicas, culturales, educativas y políticas. Si se toma como ejemplo a Colombia, se visualiza que los rellenos sanitarios son de vital importancia para la gestión de residuos, pues se recicla apenas el $17 \%$ y no se considera el tratamiento biológico y térmico. En cambio, países como Alemania y Holanda utilizan los rellenos sanitarios en una proporción del 1\%. Es decir, el 99\% de los residuos son aprovechados en reciclaje y procesos térmicos.

En Noruega la basura doméstica es utilizada como combustible, principalmente en calefacción. Este proceso se desarrolla en la planta Klemetsrud. La energía que se obtiene de estos residuos tiene una proporción equivalente de 4 a 1 con los combustibles tradicionales. Los costos de transporte son compensados con su capacidad energética. Esta planta tiene como fin producir electricidad a través de turbinas que son conducidas a domicilios e instituciones públicas. Inclusive algunas ciudades de Reino Unido llevan su basura a la ciudad de Oslo, la cual recibe bonificación económica por ello.

Otra forma de uso de los RSOU en Noruega es a través del biogás, que es un medio de alimentación para el transporte, pues es usado como biocombustible. Así $2 \mathrm{Kg}$ de RSOU puede producir 1 litro de combustible (Price, 2013). Prácticas similares se llevan a cabo en Holanda, en donde prima la economía circular para evitar el depósito de desechos en menor porcentaje posible. Sus principios son el procesamiento, obtención de energía y reciclaje, usados en la construcción. La prevención es su principal herramienta en la gestión de residuos. Para que todo ello se lleve a cabo es imprescindible políticas educativas fuertes y atractivos incentivos económicos (Ossa, 2018).

En el ámbito escolar, los estudiantes perciben a la basura como un problema, ya que es inevitable la generación de residuos orgánicos. En centros educativos su caracterización permite conocer su potencialidad en cuanto a reciclaje o aprovechamiento. Esto puede ser fuente de ahorro, obtención de beneficios económicos, disminución de volumen de basura y espacio y aumento de la vida útil de rellenos sanitarios.

Cerca del $40 \%$ al $50 \%$ de los residuos en escuelas son orgánicos, se producen alrededor de $0,134 \mathrm{Kg} /$ día de residuos alimenticios. En algunas escuelas de Sao Paulo se llega a tener una 
proporción de hasta el $90 \%$ de los mismos. Estos materiales pueden ser usados a beneficio de la misma institución en jardines y huertos, como reemplazo de fertilizantes, dando como resultado el mejoramiento de las propiedades, estructura y retención de agua en el suelo y estabilidad en los nutrientes. Con esta práctica se puede disminuir el volumen de basura en 4 toneladas al mes. El compostaje genera un impacto positivo en la disminución de GEI con una reducción del 49,86 Kg.de $\mathrm{CO}_{2}$ equivalentes/tonelada de residuos a nivel doméstico. Es así que, gran cantidad de escuelas en Sao Paulo utilizan el compostaje como herramienta práctica para la educación, conjuntamente con la conceptualización, afectividad y responsabilidad ambiental (Ricci, 2016, p. 13).

En el Centro de Investigación y de Estudios Avanzados (CINVESTAV), México, se realizó un muestreo de la basura durante 3 años, y se determinó que el $48 \%$ de la misma corresponde a materia orgánica. En la Universidad Iberoamericana de México se obtuvo una cifra similar: 52\% de materia orgánica factible para composteo (Ruíz, 2012, p. 95).

Una vez realizada la caracterización, se desarrolló un programa ambiental a través de talleres sobre reciclaje y separación, técnicas para compostaje, eliminación de residuos y divulgación de resultados. La materia orgánica fue aprovechada principalmente en el compostaje. A pesar de las deficiencias tecnológicas, infraestructura y personal se obtuvo un ahorro de aproximadamente $\$ 5000$ solo en el año 2003. Inclusive una disminución del volumen de basura en un 67\% (Maldonado, 2006, p. 64).

Para que este tipo de proyectos se lleve a cabo es necesario incrementar campañas de conciencia ambiental para todos sus integrantes. El principal problema es la falta de separación desde la fuente, cultura ambiental y el desvío de la responsabilidad hacia los administradores de las instituciones. Involucrar a las personas generadoras de basura, en la separación y utilización de los residuos incrementa la conciencia ambiental.

Los biocombustibles de segunda generación son otra alternativa para la biomasa lignocelulósica, que representa una gran cantidad de residuos orgánicos constituidos por celulosa, hemicelulosa y lignina; como los residuos de tipo forestal y alimenticio (MuellerLanger et al., 2013, p. 113). Sus principales representantes son el bioetanol y el biodiésel, cuya producción mundial se muestra en la Figura 6. 


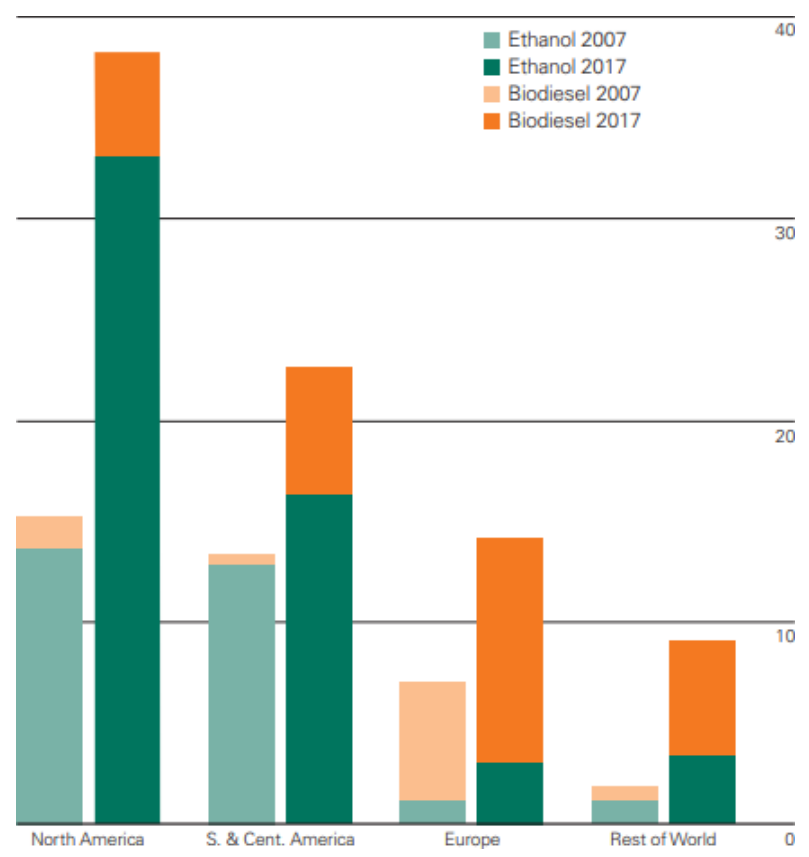

Figura 6. Producción mundial de biocombustibles (millones de toneladas equivalentes de petróleo). Fuente: (BP Global, 2018).

Los biocombustibles han ganado participación en el mundo desde 2007, como potencial reemplazo de la gasolina. El bioetanol en Sur y Centro América no ha sufrido un aumento significativo durante los años 2007 a 2017, al igual que en Europa y el resto del mundo. En Norteamérica su producción en 2007 constituyó más del doble en 2017. La participación del biodiésel se ha mantenido superior que el bioetanol durante los años de estudio.

La producción de biodiésel se lleva a cabo a través de procesos de esterificación, cuyos productos son el éster y el agua. El hidrocarburo (R') del alcohol reemplaza al hidrógeno del ácido carboxílico $(\mathrm{COOH})$ como se muestra en la Figura 7.

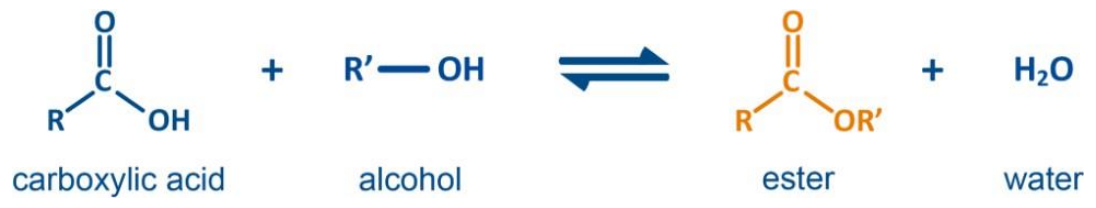

Figura 7. Producción mundial de biodiésel. Fuente: (Matthey, s.f.).

La tecnología de esterificación permite obtener ésteres no ácidos a través de una reacción del ácido carboxílico líquido con el alcohol (Matthey, s.f.).

La producción de bioetanol se realiza a través de la fermentación anaerobia de azúcares. Por lo general, se requiere de tratamientos como la hidrólisis enzimática para la liberación de azúcares simples presentes en la materia prima. Como resultado se obtienen monosacáridos fácilmente asimilables por levaduras, microorganismos que a través del proceso de fermentación transforman azúcares simples en etanol como se muestra en la Figura 8 (Kanal, s.f.) 


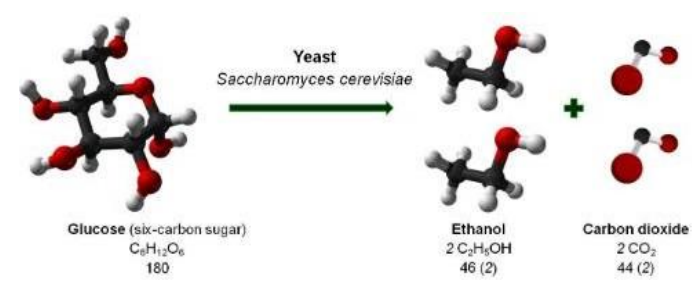

Figura 8. Producción mundial de bioetanol. Fuente: (Kanal, s.f.).

Las cáscaras de frutas como plátano, papaya, mango, piña, etc., son una buena opción para obtener bioetanol, debido a su alta composición de azúcares y carbohidratos. Los desechos producidos corresponden aproximadamente al 50\% de su peso (Jahid et al., 2018, p. 2). Estos residuos están presenten en la composición de la basura urbana, al igual que desechos vegetales, tubérculos, etc.

El metanol es considerado como otro biocombustible, ya que puede generarse a través de residuos orgánicos, aguas residuales, heces, etc. Además es un subproducto de la fermentación de bebidas alcohólicas. Es usado como aditivo a la gasolina, al igual que el bioetanol. Puede ser obtenido a partir del metano, producto del gas natural. Las desventajas en su producción es que solo presentan factibilidad económica a nivel industrial, además de peligros de toxicidad en su manipulación (Leiva, 2011).

La EA está ligada a la practicidad y la mejor manera de hacerlo es a través de los desechos, con los que convivimos a diario, debido a su fácil alcance y aplicación. El compostaje es la mejor forma de afianzar los conocimientos teóricos y crear conciencia como consumidores. El contacto con los problemas motiva propuestas e ideas de cambio, permite la inclusión de los niños en actividades sencillas que crean experiencias y conocimiento sobre la realidad de la basura.

\section{Conclusiones}

La formación educativa es un proceso que está en constante cambio debido al dinamismo de la naturaleza. Desarrollar el aspecto práctico a través de proyectos que puedan realizarse de forma sencilla con alcance en la cotidianidad, consolida el empoderamiento hacia un nuevo estilo de vida, consciente, crítico y capaz de ver la realidad y proponer soluciones a conflictos ambientales.

La educación ambiental debe realizarse desde la etapa inicial, involucrando a los niños y haciéndoles sentir parte del medio, en esta etapa es más factible captar su atención a través de una interacción, visualización, excursión y ejecución de proyectos gracias a la percepción de su entorno y sus problemas adquieren autonomía para tomar decisiones y emprender acciones, ideas o proyectos. Los niños actúan como promotores de cambio con influencia a las personas adultas y directamente a sus padres. Cada persona es diferente con inteligencias y personalidades diversas, por lo tanto, la metodología no debe ser estática, debe asumir una posición creativa con varias alternativas para asegurar la comprensión de todos.

Las TIC son medios de sensibilización y transmisión de información ambiental, complemento de la EA. Son instrumentos que deben estar acorde a las necesidades y características de grupos objetivo. Son oportunidades para promover el diálogo y la experiencia sin fronteras y de una forma más rápida. Las más importantes corresponden a los medios audiovisuales como televisión, videos principalmente, pues está demostrado que 
tiene mayor efectividad en los niños. Por otro lado, las redes sociales son usadas por casi todas las personas diariamente y varias veces en el día, pueden ser una oportunidad para difundir información, pero a través de entidades oficiales que tengan respaldo bibliográfico, pues se puede generar posiciones negativas o inciertas, que no aporten con ningún beneficio a la sociedad.

Gran cantidad de residuos son dispuestos en rellenos sanitarios para permanecer allí por largos periodos, pero estos pueden usarse como insumo para las tecnologías limpias, que causan un impacto mínimo al ambiente. Se puede ampliar la vida útil de estos desechos, a través de su conocimiento y caracterización. Una de las prácticas más sencillas y que se pueden llevar a cabo en instituciones educativas es el compostaje, en donde se aprovecha la gran cantidad de materia orgánica para mejorar las condiciones del suelo. Estas actividades permiten la resolución y acercamiento de problemas cotidianos en los niños, que son los mejores receptores de información. Así, ellos se sienten parte de la solución y con capacidad de replicarlo fuera de su entorno educativo. 


\section{Bibliografía}

Acosta, V. (2013). Las redes sociales como herramienta para la educación. Journal Technology, 12(1), 55-65.

Boeve, J., Gericke, N., Olsson, D., y Berglund, T. (2015). The Effectiveness of Education for Sustainable Development. Sustainability, 7, 15693-15717.

BP Global. (2018). Biofuels production. Recuperado el 15 de febrero de 2019, de https://www.bp.com/en/global/corporate/energy-economics/statistical-reviewof-world-energy/renewable-energy/biofuels-production.html

Calvachi, V., \& Navarro, N. (2013). Evaluación Ambiental de la Incineración de los residuos orgánicos biodegradables de los residuos sólidos urbanos. (Tesis de grado). Escuela Politécnica Nacional, Quito.

Carvajal, A. (2014). Las TIC, la educación y un ambiente sustentable y sostenible. Recuperado el 13 de febrero de 2019, de OEI: https://www.oei.es/historico/divulgacioncientifica/?Las-TIC-la-educacion-y-unambiente

Castillero, O. (2017). ¿Por qué maduran antes las chicas que los chicos? Recuperado el 18 de febrero de 2019, de Psicología y Mente: https://psicologiaymente.com/personalidad/por-que-maduran-antes-chicas-quechicos

Castillo, R. M. (2010). La importancia de la educación ambiental ante la problemática actual. Educare, 14(1), 97-111.

Cervantes, M. (2015). Educación ambiental en los medios de comunicación. Ciencia UANL(74), 16-19. Obtenido de Ciencia UANL: http://cienciauanl.uanl.mx/?p=4439

Corraliza, J. (14 de septiembre de 2016). ¿A qué edad debería comenzar la educación ambiental y quién tendría que impartirla? Recuperado el 13 de febrero de 2019, de HUFFPOST: $\quad$ https://www.huffingtonpost.es/2016/09/14/ninos-educacionreciclaje n $11989524 . \mathrm{html}$

Díaz, D., Castilo, L., y García, P. D. (2014). Educación ambiental y primera infancia: Estudio de caso Institución Educativa Normal Superior y Fundación Educadora Carla Cristina del Bajo Cauca. (Tesis de grado). Universidad de Antoquia.

EMGIRS EP. (2017). Residuos orgánicos de Quito serán aprovechados mediante

lombricultura. Recuperado el 11 de febrero de 2019, de https://www.emgirs.gob.ec/index.php/noticiasep/340-residuos-organicos-dequito-seran-aprovechados-mediante-lombricultura

FECYT. (2018). Encuesta de Percepción Social de la Ciencia y la Tecnología en España. Recuperado el 14 de febrero de 2019, de https://icono.fecyt.es/informes-ypublicaciones/percepcion-social-de-la-ciencia-y-la-tecnologia-en-espana

Gädicke, J., Ibarra, P., y Osses, S. (2017). Evaluación de las percepciones medioambientales en estudiantes de enseñanza media de la ciudad de Temuco, Región de La Araucanía. Scielo, 43(1), 107-121. 
Garza, 0. (2003). Influencia de laducación ambiental en la formación del estudiante de la preparatoria No. 13 de la U.A.N.L. (Tesis de posgrado). Universidad Autónoma de Nuevo León, México.

Graziani, P. (2018). Economía circular e innovación tecnológica en residuos sólidos: Oportunidades en América Latina. Recuperado el 19 de febrero de 2019, de SCIOTECA: http://cdi.mecon.gov.ar/bases/docelec/az4041.pdf

INEC. (2016). Estadística de Información Ambiental Económica en Gobiernos Autónomos Descentralizados Municipales. Recuperado el 15 de enero de 2018, de https://bit.ly/2IL4aY7

INEC. (2017a). Módulo de Información Ambiental en Hogares. Recuperado el 14 de febrero de 2019, de http://www.ecuadorencifras.gob.ec/documentos/webinec/Encuestas Ambientales/Hogares/Hogares 2017/DOC TEC MOD AMBIENTA L ENEMDU\%202017.pdf

INEC. (2017b). Tecnologías de la Información y Comunicación. Recuperado el 14 de febrero de 2019, de http://www.ecuadorencifras.gob.ec/documentos/webinec/Estadisticas Sociales/TIC/2017/Tics\%202017 270718.pdf

ISWA. (2013). ISWA Guidelines: Waste to Energy in Low and Middle Income Countries. Obtenido de http://www.waste.ccacoalition.org/document/iswa-guidelineswaste-energy-low-and-middle-income-countries

Jahid, M., Gupta, A., \& Kumar, D. (2018). Production of Bioethanol from Fruit Wastes (Banana, Papaya, Pineapple and Mango Peels) Under Milder Conditions. Journal of Bioprocessing \& Biotechniques, 8(3), 1-11.

Jaramillo, G., \& Zapata, L. (2008). Aprovechamiento de los residuos sólidos orgánicos en Colombia. (Tesis de posgrado). Universidad de Antoquia.

Johnson, M., y Valdiviezo, A. (2011). TIC y medio ambiente. Recuperado el 13 de febrero de 2019 , de Newsletter: https://www.cepal.org/socinfo/noticias/paginas/9/30389/newsletter14.pdf

Kanal, S. (s.f.). Bioenergy Research Group. Recuperado el 22 de febrero de 2019, de http://www2.hawaii.edu/ khanal/fungal/biofuels.html

Leiva, C. (2011). La utilización del Metanol como Biocombustible. Realidad y reflexión, (31).

MAE. (2017). Estrategia Nacional de Educación Ambiental. Recuperado el 23 de enero de 2018, de http://www.ambiente.gob.ec/wpcontent/uploads/downloads/2017/11/5-Estrategia-Nacional-deEducacio\%CC\%81n-Ambiental.pdf

Maldonado, L. (2006). Reducción y reciclaje de residuos sólidos urbanos en centros de educación superior: Estudio de caso. Ingeniería, 10(1), 59-68.

Matthey, J. (s.f.). Esterification technology. Recuperado el 22 de febrero de 2019, de https://matthey.com/products-and-services/chemical-processes/coretechnologies/esterification-technology

Ministerio de Educación. (2016). Guía Introductoria a la metodología TINI. Recuperado el 18 de febrero de 2019, de https://educacion.gob.ec/wpcontent/uploads/downloads/2017/11/metodo-TINI.pdf 
Mueller-Langer, F., Majer, S., y Perimenis, A. (2013). Biofuels: A Technical, Economic and Environmental Comparison. Springer, 110-137.

Novo, M. (2009). La educación ambiental, una genuina educación para el desarrollo sostenible. Revista de educación, 195-210.

Olsson, D., y Gericke, N. (2015). The adolescent dip in students' sustainability consciousness-Implications for education for sustainable development. The Journal of Environmental Education, 47(1), 35-51.

Ossa, F. (2018). Lecciones de Holanda en manejo de agua y residuos. Recuperado el 19 de febrero de 2019, de Semana: https://www.semana.com/vidamoderna/multimedia/las-lecciones-de-holanda-en-manejo-de-agua-residuospara-enfrentar-el-cambio-climatico/441610-3

Palavecinos, M., Amérigo, M., Ulloa, J. B., \& Munoz, J. (2016). Preocupación y conducta ecológica responsable en estudiantes universitarios: estudio comparativo entre estudiantes chilenos y espanoles. Psychosocial Intervention, 25, 143-148.

Pedroso, W., y Menéndez, Y. (2017). Vinculación de adolescentes con la gestión ambiental en comunidades costeras de Cuba, a través de la educomunicación y el audiovisual participativo. Revista de Comunicación, 16(1), 147-165.

Pol, E., y Castrechini, A. (2013). ¿Disrupción en la educación para la sostenibilidad? Revista Latinoamericana de Psicología, 45(3), 335-349.

Price, M. (2013). Cómo Noruega convierte basura en combustible ecológico. Recuperado el 19 de febrero de 2019, de BBC Mundo: https://www.bbc.com/mundo/noticias/2013/09/130923 ciencia noruega basur a energia ng

Ricci, M. (2016). A handbook for schools on organic waste management.

Romero, A. (2010). Aprovechamiento de la biomasa como fuente de energía alternativa a los combustibles fósiles. .R.Acad.Cienc.Exact.Fís.Nat, 104(2), 331-345.

Ruíz, M. (2012). Caracterización de residuos sólidos en la Universidad Iberoamericana, Ciudad de México. Revista internacional de contaminación ambiental, 28(1). Obtenido de http://www.scielo.org.mx/scielo.php?script=sci arttext\&pid=S0188$\underline{49992012000100008}$

Ruiz, R. (2016). Las redes sociales como herramienta para la Educación Ambiental.

Recuperado el 13 de febrero de 2019, de Comunidad Ism: http://www.comunidadism.es/blogs/las-redes-sociales-como-herramienta-parala-educacion-ambiental

Salinas, H., Herrera, S. D., Álvarez, D., y Marquéz, A. (2016). Sustentabilidad y educación ambiental con el uso de las TIC'S y currículo. ResearchGate(1), 2997-3007.

Vargas, J. P., Buzón, J., Vergara, D., y Molina, E. (2011). Reducción de los Residuos Sólidos Orgánicos en Colombiapor medio del Compostaje Líquido. INGENIARE(11), 37-44.

Vázquez, Á., y Manassero, M. (2005). Actitudes de los jóvenes en relación con los desafíos medio-ambientales. Infancia y Aprendizaj, 28(3), 309-327. 
Zsóka, Á., Marjainé, Z., Széchy, A., y Kocsis, T. (2013). Greening due to environmental education? Environmental knowledge,attitudes, consumer behavior and everyday pro-environmental activities of Hungarian high school and university students. Journal of Cleaner Production, 48, 126-138.

Zúñiga, C. (2004). Aula virtual: nueva herramienta para la Educación Ambiental. Revista Biocenosis, 18, 38-42. 


\section{Autores}

ALISSON YÉPEZ-CHÁVEZ es estudiante de la Universidad UTE, en Quito. Se encuentra culminando la carrera de Ingeniería Ambiental y Manejo de Riesgos Naturales.

Actualmente está desarrollando su tesis sobre obtención de bioetanol a partir de residuos de cáscaras de papa, mediante la utilización de enzimas celulolíticas. Sus principales temas de investigación incluyen el aprovechamiento de residuos sólidos, tecnologías limpias y biocombustibles.

FAUSTO VITERI-MOYA obtuvo su título de Doctor en Ingeniería Química y del Medio Ambiente por el Departamento de Ingeniería Química y Tecnologías del Medio Ambiente de la Universidad de Zaragoza (España) en 2017. Obtuvo el título de Magister en Sistemas de Gestión Ambiental en la Universidad de las Fuerzas Armadas ESPE en 2013 y el título de Ingeniero Agropecuario en la misma universidad en 2008.

Actualmente es profesor titular de la Facultad de Ciencias de la Ingeniería e Industrias en la Carrera de Ingeniería Ambiental y Manejo de Riesgos Naturales de la Universidad UTE. Es líder del grupo de investigación "Grupo de Protección Ambiental". Entre sus trabajos más relevantes se encuentran publicaciones en el proceso de pirolisis de compuestos oxigenados y azufrados, centrado en la identificación y cuantificación de hidrocarburos aromáticos policíclicos (HAP's), y procesos meteorológicos. Con un $95 \%$ de publicaciones en artículos indexados en el Journal Citation Reports (JCR), en revistas Q1. Además, ha presentado sus trabajos en más de una docena de congresos internacionales incluyendo su participación en el "8th European Combustion Meeting 2017". 